\title{
FITNESS CONSEQUENCES OF CHOOSY OVIPOSITION FOR A TIME-LIMITED BUTTERFLY
}

\author{
Patricia Doak, ${ }^{1,4}$ Peter Kareiva, ${ }^{2}$ And Joel Kingsolver ${ }^{3}$ \\ ${ }^{1}$ Institute of Arctic Biology, University of Alaska, Fairbanks, Alaska 99775 USA \\ ${ }^{2}$ The Nature Conservancy, 4722 Latona Avenue NE, Seattle, Washington 98105 USA \\ ${ }^{3}$ Department of Biology, CB-3280, University of North Carolina, Chapel Hill, North Carolina 27599-3280 USA
}

\begin{abstract}
For the majority of insects, a female's choice of oviposition site(s) greatly influences both the success of individual offspring and her own total fitness. Theory predicts that females most strongly limited by egg number will employ greater oviposition site discrimination than those predominately subject to time limitation. The reproductive success of the butterfly Pieris virginiensis at our Connecticut, USA, field site is strongly time constrained on two fronts. First, during their three-week flight season, only $60 \%$ of days and $28 \%$ of daytime hours were suitable for flight. Second, larval survival is impacted by the rapid senescence of their spring ephemeral host plant Dentaria diphylla, with eggs laid during the first half of the flight season having approximately three times the survival chance of those laid later. Yet, on average, females choose to oviposit on only half the plants they closely inspect and fly over most ramets without any inspection. Our experiments demonstrate that the preferred host ramets confer an approximate two-fold survival advantage. Females are not choosing plants that senesce later, despite the advantage that such plants would confer. We use empirical data on female behavior and larval performance to parameterize a simulation model. Model results suggest that, despite the notable time limitation in this system, the observed level of female oviposition site preference not only increases individual larval survival, but also total female fitness. Low egg loads in this species may contribute to selection for strong host plant discrimination.
\end{abstract}

Key words: Dentaria diphylla; host plant discrimination; larval survival; oviposition; performance; Pieris; preference; time limitation.

\section{INTRODUCTION}

In the majority of holometabolous insects, adults are more mobile than larvae, making a female's choice of oviposition site critical for both the fitness of her offspring and her own inclusive fitness. This is especially true for herbivorous insects, the majority of which are moderately to extremely specialized in diet (Strong et al. 1984). For a particular insect, host plant acceptability and quality will vary among plant species, populations, and individuals; quality will also differ among plant parts and tissues of differing age (Price 1984, Strong et al. 1984).

Theory predicts that oviposition strategies of herbivorous insects will vary depending on whether a female is more limited by the time available for oviposition or the number of eggs she can lay (Levins and MacArthur 1969, Jaenike 1978, Courtney 1982, Chew and Robbins 1984, Mangel 1987). The relative strengths of these factors will differ during an individual's lifetime, as well as among populations and species. A time-limited female with many eggs may be less picky about oviposition sites, because each egg only constitutes a small proportion of her reproductive

Manuscript received 21 April 2005; revised 18 July 2005; Accepted 28 July 2005. Corresponding Editor: M. D. Eubanks.

${ }^{4}$ E-mail: ffpd@uaf.edu investment. On the other hand, females with plenty of time but few eggs are predicted to be quite picky about oviposition sites; in this case, each egg constitutes a large proportion of her potential fitness, and she has both the need and the time to choose carefully.

An implicit assumption of these models is that the choosy female garners increased fitness through her discrimination. The majority of research addressing the benefits of female preference has focused on larval performance gained through direct effects of the host plant on the larvae: higher nutritional quality of the host and/or reduced impact of plant defenses. Empirical work indicates that sometimes larval performance follows female preference (Carr et al. 1998, Fritz et al. 2000, Joachim-Bravo et al. 2001, Kessler and Baldwin 2002, Macel et al. 2002, Wise and Weinberg 2002), and sometimes it does not (Craig et al. 2000, Cronin and Abrahamson 2001, Solarz and Newman 2001, Baker and Candy 2002; also see Chew and Robbins 1984, Singer 1984, Thompson 1988, and Mayhew 1997 for reviews). However, a female may accrue other benefits through her discrimination. Her eggs and/or larvae may experience reduced predation or parasitism (Thompson 1988, Mayhew 1997, Ballabeni et al. 2001, Pierce et al. 2002, Shiojir and Takabayashi 2003) or lower intraor interspecific competition (Rausher 1979, Dempster 1983, Chew and Robbins 1984, Fletcher and Prokopy 
1991). Microsite characteristics may directly influence larval fitness (Forsberg 1987), or the female may choose sites that enhance her own survival by avoiding predators or providing her with food (Thompson 1988, Scheirs et al. 2000, 2004, Scheirs and De Bruyn 2002).

A positive relationship between female oviposition preference and larval performance does not by itself establish that female choice increases her fitness. Discrimination among host plants will decrease the rate of oviposition and expose the female to greater mortality during her extended search, thereby reducing her chances of laying her full complement of eggs. Later oviposition may also result in lower egg and larval survival (Ehrlich et al. 1975, Shapiro 1979, Cappuccino and Kareiva 1985, Weiss et al. 1988, Cushman et al. 1994, Boughton 1999). Finally, an oviposition strategy favored in some parts of an herbivore's range may be maladaptive elsewhere where time for oviposition is more limited, daily survival probability of females is low, and/or time for larval development is constrained by host plant phenology, seasonal conditions suitable for larval growth (Ehrlich et al. 1975, Weiss et al. 1988, Cushman et al. 1994, Boughton 1999), or interspecific interactions.

Here we examine the relationship between female host plant choice and offspring performance in the locally monophagous butterfly, Pieris virginiensis Edwards. Time for adult flight and oviposition is tightly constrained by seasonal climatic conditions, and a short window of opportunity exists for larval development due to senescence of its spring ephemeral host plant, Dentaria diphylla Michx (Cappuccino and Kareiva 1985). This allowed us to examine the benefits of host plant choice in a situation where time is of the essence for both completion of oviposition and successful larval development. Specifically, we sought to address the question of whether female choosiness increases overall female fitness. To do this we examined: (1) the yearly amount of time available for flight and oviposition, (2) the rate of oviposition and the degree of female choosiness, (3) larval survivorship as a function of time of oviposition, (4) larval survivorship on chosen vs. unchosen host plant individuals, and (5) the relationships between larval density, water availability, host senescence, and larval survivorship. Finally, we used the field data to parameterize a simple simulation model to examine whether observed female strategies are likely to lead to an overall fitness advantage.

\section{NATURAL History}

At our study site, Pieris virginiensis specializes on the host plant Dentaria diphylla (Cappuccino and Kareiva 1985). P. virginiensis is univoltine with adults emerging from overwintering pupae in April, just after the emergence of its host plant. Ovipositing females fly above the height of their host plant. The majority of host ramets are completely bypassed with no deviation in flight path. Occasionally a female descends to inspect a plant by fluttering just above a host leaf, and then she either immediately alights to oviposit or resumes flight. Eggs are laid singly on the undersides of host plant leaves. In May, larvae hatch and complete development in approximately three weeks, at which time fifth-instar larvae pupate and enter diapause until the following spring. D. diphylla foliage is beginning to senesce by mid- to late May, and in June, aboveground growth of Dentaria is finished for the season. When $P$. virginiensis adults emerge in April and early May, the forest canopy is open, but it closes quickly during the month of May, leaving the forest floor well shaded. Because $P$. virginiensis requires almost full sun for flight (Cappuccino and Kareiva 1985), it has a narrow window of opportunity for flight and oviposition between the cold and inclement weather of early spring and the closing of the forest canopy.

\section{Study Site}

This study took place in a deciduous forest on Totoket Mountain, a limestone ridge, owned and managed by the New Haven Water Company, in New Haven County, Connecticut (CT), USA. Our observations and experiments were concentrated in two sub-sites. Much of the work was done at the Creek Site (Cappuccino and Kareiva 1985), which contained a dense stand of Dentaria diphylla both directly beside and in the forest surrounding a small intermittent stream. The Forest Site lay $\sim 400 \mathrm{~m}$ to the north of the Creek Site and encompassed a forest area of similar composition, but with more widely dispersed patches of the host plant.

\section{Methods}

Our study spanned three years (1986-1988) and involved a wide variety of observations, experiments, and a computer simulation.

\section{Activity censuses}

We made observations of flight activity to get a general idea of the time available for flight each season. Each season, we designated stations (7 in 1986 and 8 each in 1987 and 1988) from which we took visual censuses of the number of adults in flight. We spent 1 min at each station, doing two circuits of all stations during a census period in both 1986 and 1988. In 1987 one circuit of the stations comprised a census period. We took censuses roughly every hour when we were in the field unless conditions were such that absolutely no flight was occurring (e.g., sleet, low temperatures). We recorded flight activity from 27 April to 13 May in 1986, from 23 April to 13 May in 1987, and from 17 April to 14 May in 1988. All censuses were done along the same stretch of bank at the Creek Site.

We used climatological data from Bradley Field, Hartford, CT (National Climate Data Center, Asheville, North Carolina, USA; Cappuccino and Kareiva [1985] found conditions at this station to best match those of the field site) to examine flight activity in relation to 
regional temperature and cloud cover for both the 1986 and 1987 field seasons. Climate observations were taken at 3-h intervals; we matched these with corresponding activity censuses to make our comparisons. Sky cover was rated in tenths on a scale of 1-10.

\section{Female flight and oviposition}

To examine female plant choice and oviposition behavior, we followed individual females and recorded their activities. Observations began when we encountered females in flight; females were not manipulated before or during the observation periods. In 1986 we followed 12 females and recorded the number of eggs laid per time. The rate of oviposition is impacted by several distinct factors: a refractory period between egg maturation, a female's propensity to disperse, and host plant discrimination. To better understand oviposition patterns, in 1987 we followed 28 females and recorded each time a female investigated a plant for oviposition and laid each egg, along with a time stamp. This allowed us to calculate the rate at which plants were investigated, the rate of oviposition, and the number of ovipositions per plant investigated. A female that investigates a plant for oviposition is probably ready to oviposit, and plants rejected at this stage appear to be actively selected against. Unfortunately, the time between investigations remains confounded by latency, dispersal, and any host plant discrimination that occurs prior to close investigation.

\section{Natural quadrats}

To examine natural survival probabilities and survival as a function of date of oviposition, in 1987 and 1988 we marked 57 and 67 unmanipulated $0.25-\mathrm{m}^{2}$ quadrats, respectively. All of these quadrats were at the main Creek Site. We recorded the number of ramets in each quadrat. In 1987 we searched quadrats for eggs and larvae every 2-5 d between 30 April and 1 June. In 1988 we searched quadrats every $1-2 \mathrm{~d}$ from 18 April to 18 May and then at least every third day until 4 June. At each census, the number of eggs and larvae present and the instar of each larva were recorded. These data were used to calculate survival of naturally occurring eggs to instar IV.

Fifth-instar larvae are mobile, making it impossible to assess survival through this terminal stage. Therefore, we used presence to the fourth instar as our measure of survival. First- through fourth-instar larvae seldom leave their host plant unless it is killed or completely senesces. Cappuccino and Kareiva (1985) note that larvae younger than instar IV have almost no chance of successfully moving between host ramets. Therefore, those larvae that disappeared prior to the fourth instar were recorded as dead.

\section{Plant choice and larval survival}

Because ovipositing $P$. virginiensis often pass up what appear to be suitable host ramets, we designed an experiment to look at possible differences between plants picked for oviposition ("egg plants") and those that are not picked ("empty plants"). We used larval survival as a bioassay for differences between these two classes of plants.

In 1987 we marked 40 pairs of egg and empty plants at the Forest Site; three of the egg plants and one of the empty plants were subsequently destroyed and dropped from the experiment. The paired plants were 2-6 m apart, controlling for local environmental variation. All egg plants were selected from within 0.25$\mathrm{m}^{2}$ quadrats containing two or more eggs; the actual focal plant was picked as the plant bearing the most eggs within the quadrat. We selected as empty plants those that had no eggs or larvae present and were in a quadrat containing one or fewer eggs. The number of plants per quadrat varied, but the treatment means did not differ (mean \pm SE; with eggs, $9.60 \pm 0.86, n=$ 40; without eggs, $9.56 \pm 0.94, n=40$ ).

In 1988 we expanded the experiment to include 66 pairs of egg and empty plants at the Creek and Forest Sites. In that year, egg plants were chosen purely on the basis of whether or not they bore at least one egg. Empty plants were within $20-100 \mathrm{~cm}$ of their paired egg plant to better control for microhabitat variation, and they were visually paired for size. We also cleared all other $D$. diphylla ramets within a $10 \mathrm{~cm}$ radius around each experimental plant.

In both 1987 and 1988, we collected eggs from throughout the woodland that contained our study population and reared them to the first instar. We removed all naturally occurring eggs and larvae from the focal egg plants on the day that we introduced the "study" larvae; eggs later found on these plants were also removed. Each egg and empty plant was inoculated with one first-instar larva.

Larvae were introduced to both plants in a pair on the same day; these introductions occurred on 9-16 May in 1987 and on 14-16 May in 1988. The introductions were later in 1988 due to later phenology that year. We censused the plants for presence or absence of larvae on 15, 19, 24, 29 May and 1 June 1987, at which time the majority of larvae had either disappeared or reached instar V. In 1988 we censused the plants on 19, 24, 26, 28, 30 May, 2 and 4 June; in this year, even on 4 June, many of the larvae had only reached instar IV. We then compared survival from instar I to instar IV between plants that had and had not been chosen for oviposition.

\section{Plant color}

Because host plant senescence has been suggested as a possible factor in determining larval survival, we sought to examine whether eggs were naturally laid on plants that senesced less rapidly and whether larvae survived better on these plants. In 1988 we categorized the condition of plants in the plant choice experiment by matching their leaf color to that of an eight-point 
color scale that ranged from yellow to green (Grumbacher Color Compass, New York, New York, USA). This was done once on 28 May, which was midway through the experiment; $33 \%$ of those larvae surviving to instar IV had reached that life stage before this date. Ten plants in each treatment class (egg and empty) were dropped from this analysis because no larvae were recorded after the date of transplant. Five additional plants were no longer present, due to consumption or complete senescence, on 28 May. This left us with 54 egg plants and 53 empty plants.

\section{Larval density and host plant quality}

These experiments, performed in 1986 and 1987, examined two possible factors affecting larval survival. First, we looked at the effect of high larval density on survival, and secondly we investigated the effect of watering host plants. If host plants are a limited resource, then boosting density could impact survivorship. Likewise, watering could affect survivorship by improving plant condition.

In 1986 we manipulated larval density in $4-\mathrm{m}^{2}$ quadrats, each surrounded by a 1-m swath cleared to reduce the possibility of larval immigration. The quadrats were arrayed into 10 triplets. Each triplet included a quadrat with: (1) one naturally occurring larva (low-density control), (2) one transplanted larva (low density), and (3) four transplanted larvae (high density). The number of host ramets per quadrat ranged from 5 to 50 ramets per quadrat $(20.7 \pm 4.81)$. We equalized host plant density within each triplet by removing ramets. Firstinstar larvae were transferred to the experimental quadrats between 12 and 23 May, and larval survival was followed through 2 June.

As a separate experiment, in 1986 we selected 40 pairs of plants that were matched with respect to size and were within $0.5 \mathrm{~m}$ of one another. Half of these pairs were located directly beside the creek, while the other half were on the bank well above the level of the creek. One plant of each pair was watered every other day (1 L of water) from 29 April to 31 May, while the other was left as a control. First-instar larvae were transplanted to each plant between 6 and 10 April and larval survival and instar were censused until 2 June.

In 1987, we set up 21 sets of three $0.25-\mathrm{m}^{2}$ quadrats to examine the influence of both larval density and watering on larval survival. Quadrats within a triplet were 1-3 m apart. Each triplet included a quadrat with: (1) a single larva introduced to a randomly picked plant (low density), (2) four larvae introduced to randomly chosen plants (thus, a single plant could receive more than one larva; high density), (3) four larvae introduced as in treatment 2 , but in which each plant receiving a larva was watered every second day (high density, watered). Four larvae per quadrat is a higher than average density (natural quadrats that contained eggs had 2.65 \pm 0.37 and $1.88 \pm 0.30$ eggs for 1987 and 1988, respectively). The plants within quadrats were thinned

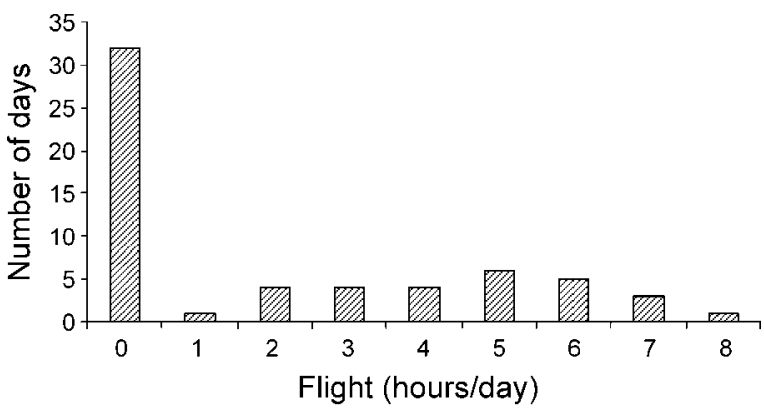

FIG. 1. The distribution of flight hours of the butterfly Pieris virginiensis, summed over the 1986-1988 seasons, used in simulation models.

so that all quadrats within a triplet had the same number of plants (range $=5,21 ; 12.29 \pm 1.19$ ). We also removed all naturally occurring eggs and larvae. The amount of water delivered was $\sim 1 \mathrm{~L}$ at the start of the experiment, but was increased to $\sim 2 \mathrm{~L}$ as the weather became hotter and drier; we did not water on rainy days. First-instar larvae were transferred to experimental quadrats from 8 to 13 May. Larval presence/ absence and instar were then censused until 1 June.

\section{Simulation}

We used a simulation model to examine the impact of time limitation on female reproductive success. The simulation model compared the reproductive fitness of individuals that followed the host plant choice and oviposition patterns we observed in our population ("choosy" females) with hypothetical "indiscriminate" females that laid eggs on all investigated hosts. Briefly, each simulation began by randomly picking from specific probability functions: the number of hours available for flight each day, the emergence date, the adult life span, and the rate at which plants are investigated for possible oviposition. Then the model separately calculated the reproductive success of a choosy and an indiscriminate female. While egg load is often a critical component of fitness estimates, many butterfly species are limited by the opportunity to lay eggs rather than their supply of eggs (Courtney and Duggan 1983, Dempster 1983, Kingsolver 1983, Courtney 1984, 1986). Given this and our focal interest in time limitation and host plant discrimination, we did not vary egg load between the two oviposition strategies.

For each day of the 17-day adult season, the number of hours suitable for flight was randomly selected with replacement from a probability distribution based on our empirical observations in 1986-1988 (Fig. 1); we found no evidence of autocorrelation in the hours of flight per day. Next, a female's day of emergence and life span were randomly selected from probability functions. Our empirical data did not provide information on the shape of emergence or survivorship curves, nor have we located these data for closely related species. 
TABLE 1. Parameter values for the simulation models used in this study investigating the choice of oviposition site and reproductive success of the butterfly Pieris virginiensis.

\begin{tabular}{ll}
\hline \hline \multicolumn{1}{c}{ Parameter } & \multicolumn{1}{c}{ Values } \\
\hline Host plants investigated/min $\dagger$ & normal distribution, $\bar{X}=0.36,95 \% \mathrm{CL}=0.22,0.55 \ddagger$ \\
Probability of oviposition for choosy $\$$ & $=0.181+0.036$ age $-0.149 \ln ($ investigations $/$ min +0.1$)$ \\
Egg load & 75 or 100 eggs/female at the start of season \\
Egg survival & $0.50,0.57,0.64 \|$ \\
Larval survival & $=\exp (0.586-0.153$ day $) /(1+\exp (0.586-0.153$ day $)) \mathbb{I}$ \\
Ratio indiscriminate : choosy survival & $0.48,0.56,0.63 \|$ \\
\hline
\end{tabular}

Note: Day refers to the day of the flight season (1 to 17) and age to female age at the time of a potential oviposition event. "Choosy" females display observed host plant discrimination when choosing sites to oviposite; "indiscriminate" females are hypothetical butterflies that oviposit on all investigated host plants.

$\dagger$ Distribution truncated at 0 and 2 investigations/min.

$\neq$ Back-transformed from $\ln ($ investigations $/ \mathrm{min}+0.1$ ).

$\S$ Bounded by 0 and 1.

$\|$ Shown are the 1988 value, mean value, and 1987 value.

II Best-fit parameter values are shown. A two dimensional 95\% CI was computed using an adaptation of the likelihood profile method (Hilborn and Mangel 1997, Kauffman et al. 2004).

However, activity censuses, albeit complicated by the influence of temperature, cloud cover, and canopy cover, did provide data on relative population size over the season. We used these data to check whether our hypothetical emergence and survivorship functions led to realistic seasonal population trends.

The emergence day for a female was then randomly picked from the distribution function. We examined a variety of lognormal emergence functions, with mean day of emergence varying from 2 to 6 days and variance varying from 1 to 6.25 . We also examined model results with uniform emergence over the 17-day flight season. Emergence function did not have a strong influence on model outcome. Therefore, we focus our reported results on the function leading to the most realistic trends in population size $\left(\mu=5, \sigma^{2}=4\right)$.

We examined two hypothetical adult survivorship curves. The first, "adult survivorship I," assumed a constant daily survivorship of 0.80 and a maximum life span of 14 days. The second survivorship curve, "adult survivorship II," assumed daily survival of 0.95 and 0.20 for the first and second weeks of life, respectively; again maximum life span was set at 14 days. Under laboratory conditions, $P$. virginiensis survive 10-21 days, skewed to shorter life spans (F. Chew, personal communication) However, Courtney (1986) notes that Pierids often have strikingly lower survivorship in the field than in the laboratory, and Kingsolver (1988) reports that mean adult life span for most studied Pierids is $\sim 3-7$ days. When combined with the best emergence function $\left(\mu=5, \sigma^{2}=4\right)$, mean adult survivorship is 4 and 8 days for the two survivorship functions, respectively.

The rate at which a female investigated host plants was randomly selected from a normal distribution based on our empirical observations (Table 1). All additional empirical estimates were fixed within a model run, and simulations were performed with a range of values. Once the number of plants investigated for possible oviposition was determined, the model separately calculated the reproductive success of choosy and indiscriminate females. We limit our comparisons to these two levels of oviposition discrimination, because we lack empirical data to estimate the shape of the relationship between survival and choosiness. Thus, our dichotomous survival estimates for larvae on chosen and unchosen host plants directly lead to our dichotomous model structure.

All females began a season with either 75 or 100 eggs (the maximum number of observed eggs in fieldand laboratory-reared individuals is $\sim 100$; F. Chew, personal communication). Choosy individuals oviposited on investigated plants as a function of female age and the rate of investigation (Table 1); this leads to a distribution of choosiness centered around the mean of 0.44 . This relationship was derived from our 1987 data. One shortcoming of these data is that the impacts of female age and the day of the season are confounded. Survival probabilities were based on data from our natural-quadrat surveys with egg survival fixed and larval survival a function of the day of the flight season on which oviposition occurred (Table 1). As choosiness decreased with female age, the model incorporated a reduction in the survival advantage of her offspring, so that eggs in excess of the number laid at a female's maximum choosiness had survival probabilities equal to that of indiscriminately laid eggs.

The hypothetical indiscriminate female oviposited on each plant that she investigated. The survival of indiscriminate offspring to instar IV was a fixed proportion of choosy survival based on the results of the plant choice experiment (Table 1); thus, it was also discounted for the day of the season.

For each set of parameters we generated 2500 flight seasons, and within each flight season we calculated the success of 100 pairs of choosy and indiscriminate females. Results were examined in two ways: pairwise comparisons between choosy and indiscriminate females and global comparisons between all choosy and all indiscriminate females across all 2500 simulations 

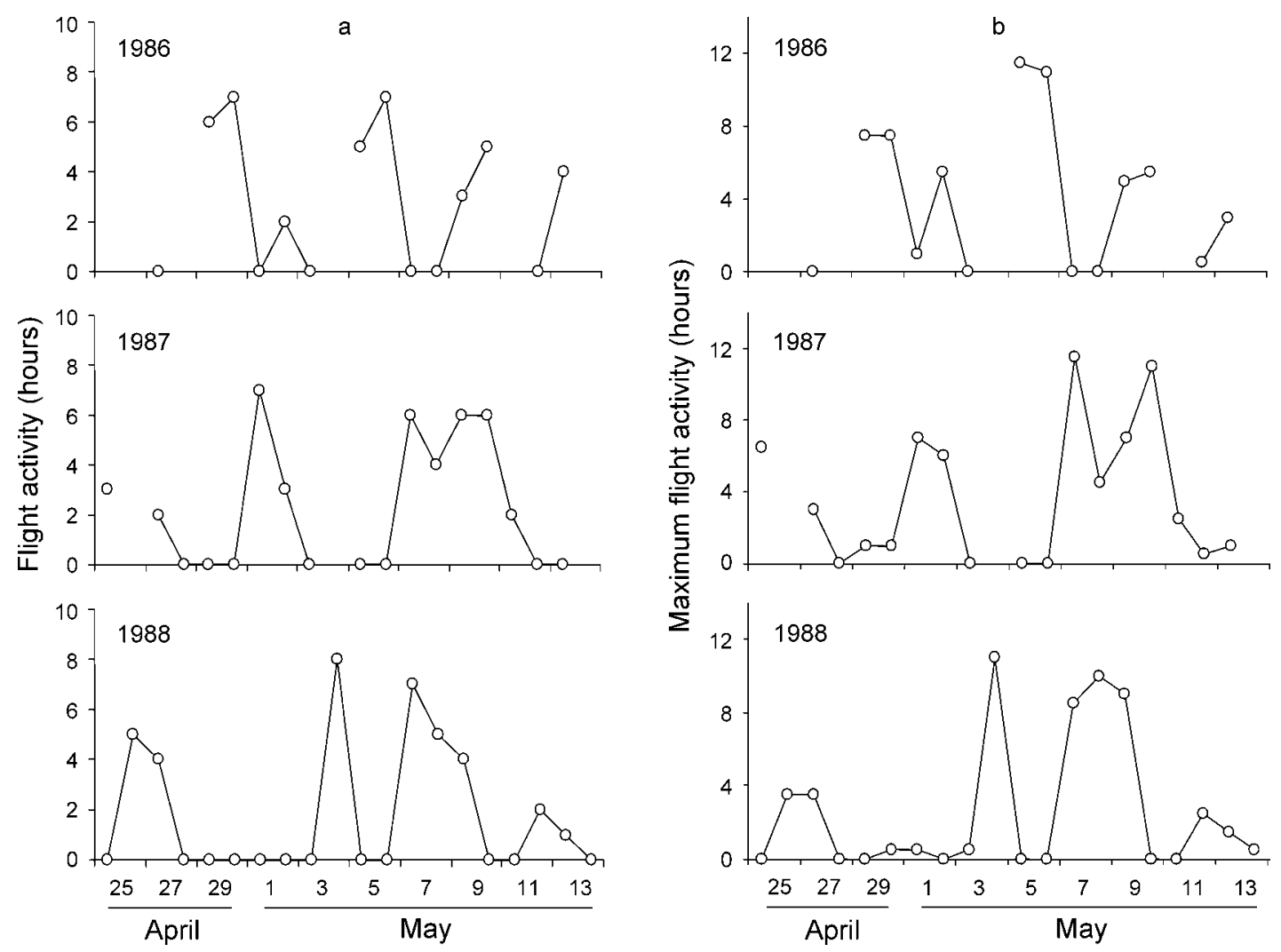

FIG. 2. Pieris virginiensis flight activity during the 1986-1988 seasons: (a) the number of hours each day when one or more adults were observed in hourly surveys, and (b) the mean of the two most populous hourly surveys. Missing data lines indicate missing data for a census day.

with a given parameter set. Within each simulated season, the median number of fourth-instar offspring was calculated for both choosy and indiscriminate females, and then a mean of these median values was computed across all 2500 simulated seasons.

\section{RESULTS}

\section{Flight activity}

In the 20 days from 25 April to 14 May, we observed 11,13 , and 12 days with at least one recorded flight in 1986, 1987, and 1988, respectively (we were unable to collect activity data during this period on five and three days in 1986 and 1987, respectively). In 1988 we observed a single butterfly on 17 April, but then no flight activity until 26 April. By mid-May, flight activity was low even on sunny days (Fig. 2a, b). Maximum flight activity occurred between 29 April and 10 May ; however, in each year, many days during this period were unsuitable for flight due to poor weather conditions (Fig. 2a). We never observed more than eight hours of flight activity in a day, and many days supported notably less than this maximum (Fig. 2a). We observed flight during 33\%, 29\%, and $23 \%$ of possible flight hours (with a maximum of 8 hours of flight/day) in the three years. There were $4.68 \pm 0.39$ hours of activity (mean \pm SE) on days when more than one individual flight was seen over the day.

As expected, temperature had a positive effect on flight activity, while cloud cover had only a marginal impact $(t=5.65, P<0.0001 ; t=-2.08, P=0.04$, respectively). The marginal impact of cloud cover is not surprising given that clear skies can be associated with very low morning temperatures as well as high midday temperatures. Also as expected, flight activity increased and then declined over the course of the flight season, resulting in a significant quadratic effect of the date on activity.

\section{Female flight and oviposition}

Of the 40 females that we observed individually, 32 laid eggs. Considering all females, a median of 0.12 eggs were laid per minute of observation time $(N=39$; Fig. 3a). In 1987 females engaged in inspection of potential oviposition sites a median of 0.37 times per minute $(N=27)$ and laid eggs on less than half of those plants inspected $(0.44 \pm 0.06, N=24$; Fig. $3 b)$. The rate of oviposition was not significantly related to the year, day (within year), or length of our observation $\left(F_{1,39}=0.91, P=0.346 ; F_{1,39}=0.07, P\right.$ $=0.798$; and $F_{1,39}=0.66, P=0.423$, respectively). 

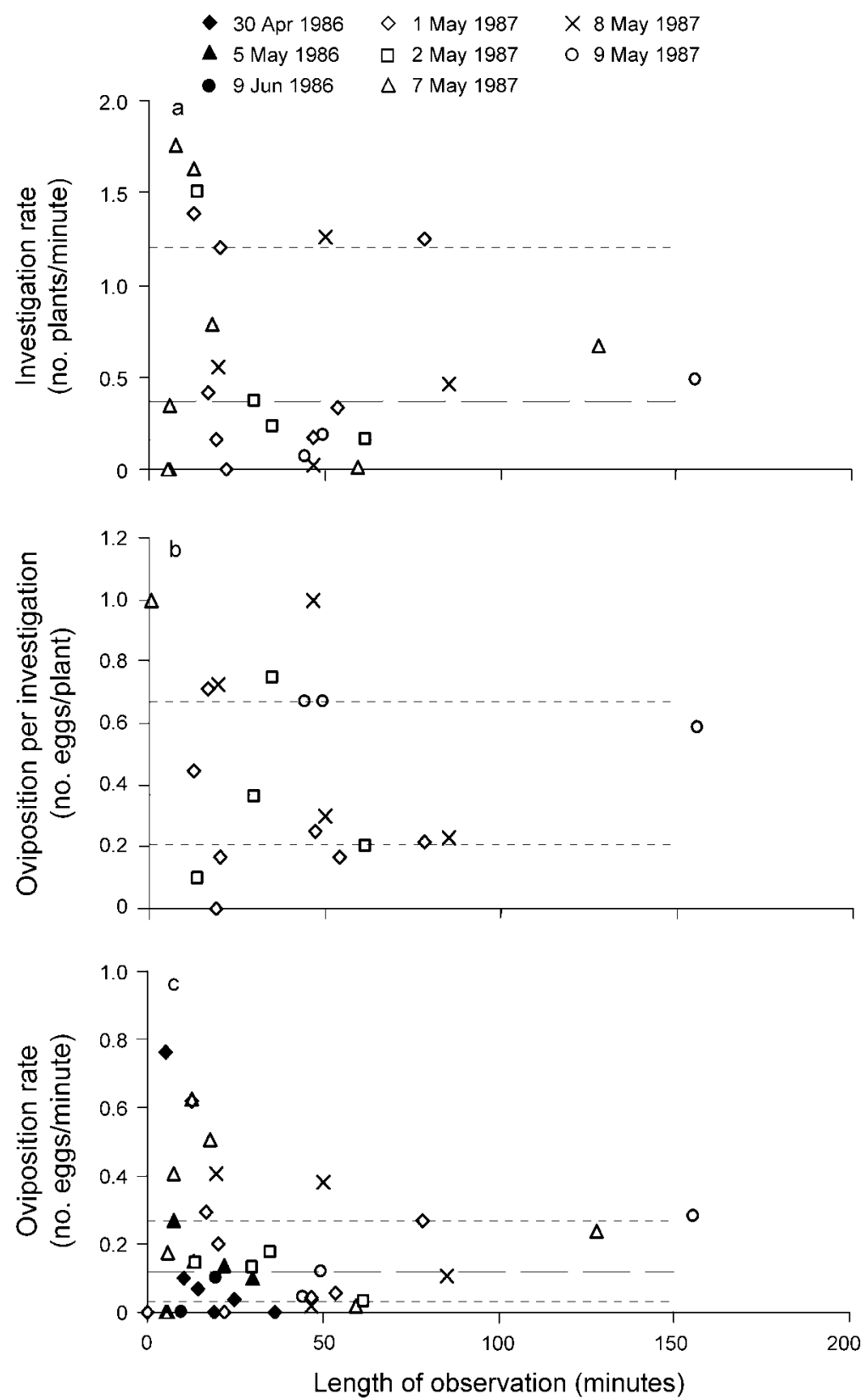

FIG. 3. Pieris virginiensis oviposition behavior: (a) the number of plants investigated for oviposition as a function of observation time, (b) the number of eggs laid per plant investigated, and (c) the number of eggs laid per minute of observation time (minutes observed $=5 \mathrm{~min}$ ). The dashed line is the median; dotted lines are the lower and upper quartiles. Investigation behavior was only recorded in 1987

Similarly, the rate of investigation of plants for oviposition was not significantly related to the day or length of our observations $\left(F_{1,26}=0.07, P=0.789\right.$, and $F_{1,26}=0.28, P=0.601$, respectively). The number of ovipositions per plant investigated increased over the season $\left(F_{1,23}=6.70, P=0.017\right)$ and decreased with the $\log$ of the investigation rate $\left(F_{1,23}=7.45, P\right.$ $=0.013$; Table 1$)$, but was not related to the length of our observations.
Natural phenology and survival

Survivorship of naturally occurring eggs to hatching was 0.64 in $1987(N=85)$ and 0.5 in $1988(N=58)$. Of those that survived to hatch, survival to instar IV was 0.41 in $1987(N=54)$ and 0.21 in $1988(N=29)$. Thus, overall survivorship from egg to instar IV was 0.26 in 1987 and 0.10 in 1988 .

To determine if the timing of oviposition impacted survival, we divided the season into two periods: "ear- 


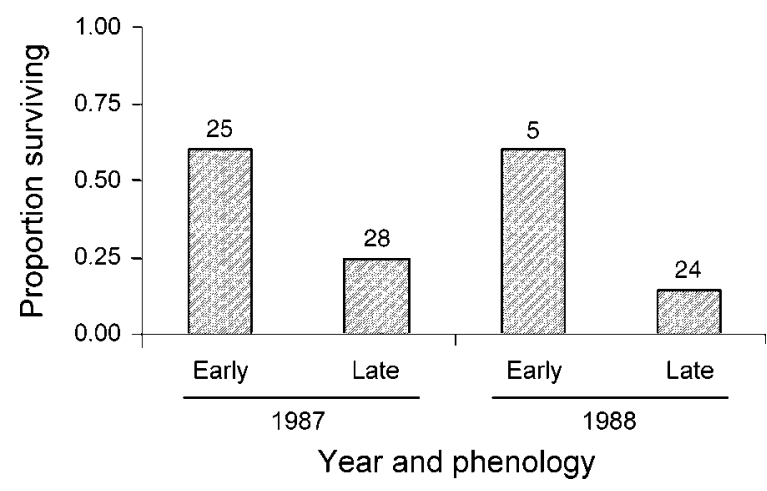

FIG. 4. Survival to instar IV of $P$. virginiensis larvae that successfully hatched in the natural quadrats. Sample sizes are noted above bars.

ly" included dates prior to 3 May and "late" included all dates from 3 May and after. Survival of the eggs to hatching did not significantly differ between times of the season, but survival to instar IV was significantly greater for eggs laid early in the season $(G=11.08$, df $=1, P<0.005$; Fig. 4). In both 1987 and 1988, $60 \%$ of early eggs survived, while only $25 \%$ and $13 \%$ (respectively) of late eggs survived.

\section{Plant choice and larval survival}

As in earlier analyses, we used presence to the fourth instar as our measure of survival. In 1988, four healthy third-instar larvae remained on the last census date and were also considered to have survived. Larval mobility introduces a further ambiguity to this data set. First, if a plant is consumed, dries out, or senesces, even early instar larvae may move and end up on other plants. Therefore, a larva may disappear from one of our census plants but survive on a neighboring plant, where we may or may not locate it. Alternatively, larvae may die on our census plants but be replaced by nonexperimental larvae. Since we are interested in the "quality" of an individual plant, following survivorship of larvae that move to adjacent plants is not informative. Therefore, larvae absent from the focal plant were recorded as dead. If a larva was not found on two consecutive censuses, it was recorded as gone and therefore dead, decreasing the chances that we mistook immigrants for our original experimental larvae.

Larval survival was close to twice as high (45\% vs. $24 \%$ ) on plants that were chosen by females for oviposition $(G=10.15$, df $=1, P<0.005)$, and the pattern was consistent between years (Fig. 5).

\section{Plant color}

We combined the eight colors into four categories: yellow, yellow/green, green, and dark green. Late season color did not differ between plants that were and were not chosen for oviposition $(G=1.30, \mathrm{df}=3, P$ $>0.10$; Fig. 6a). However, survival was higher on greener plants $(G=12.90$, df $=3, P<0.005)$, and no larvae survived on those plants that had turned yellow at the time of our census (Fig. 6b).

\section{Larval density and host plant quality}

We detected no effect of the density of larvae in a $0.25-\mathrm{m}^{2}$ quadrat on survival, as measured by survival to the instar IV (for 1986, $G=1.19$, df $=1, P>0.05$; for $1987, G=0.66$, df $=1, P>0.5$ ). In 1986 there was no effect of either plant location in relation to the creek $(G=2.26$, df $=1, P>0.05)$ or watering treatment $(G=0.09$, df $=1, P>0.5)$ on larval survival. Similarly, larval survival was unaffected by watering in 1987 ( $G=0.66$, df $=1, P>0.50)$.

\section{Simulation}

The choosy strategy led to greater variance in reproductive success among individuals experiencing the same simulated flight season (Fig. 7a). In all of our simulations, some, but never all, individuals completely failed to reproduce, and complete failure was always shared by the paired choosy and indiscriminate strategies (Fig. 7a) The within-season pattern translated into greater variance in choosy success across the 2500 flight seasons simulated for each set of parameter values (Fig. 7b). The greater variance of the choosy strategy is due to high reproductive success under favorable values for the stochastic parameters, while less favorable values led to lower offspring numbers that usually differed little between oviposition strategies. Considering the pairs of indiscriminate and choosy individuals, the choosy strategy achieved higher numbers of offspring than did the indiscriminate strategy (Fig. 8). The difference in variance between the two strategies makes it possible for a strategy to be favored in the majority of simulations and yet have lower offspring production or vice versa.

We considered two metrics for comparison of the two strategies. First we calculated the proportion of paired individuals (100 pairs per simulated flight season) where reproductive success was greater for the choosy compared to the indiscriminate individual and took the mean of these proportions across all simulations with a particular parameter set. Second, we calculated the number of fourth-instar offspring for each strategy as the mean (over 2500 simulated flight sea-

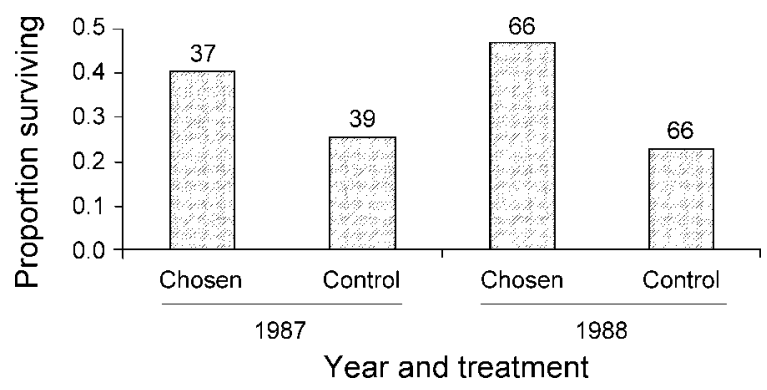

FIG. 5. P. virginiensis larval survival to instar IV in the plant choice experiment. Sample sizes are noted above bars. 

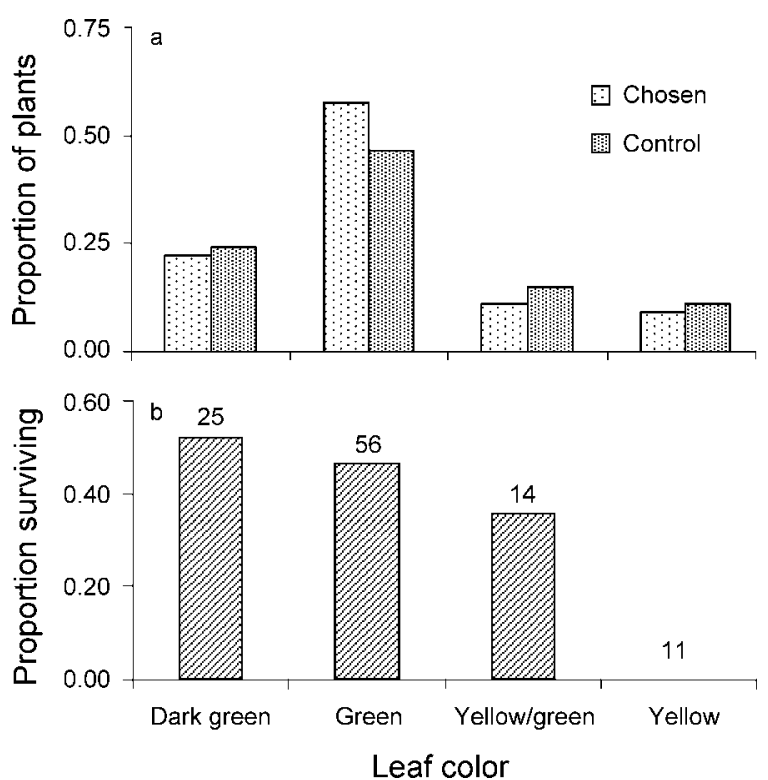

FIG. 6. Leaf color midway through the larval development period for the 1988 plant choice experiment: (a) proportion of chosen and control plants in each color class and (b) proportion surviving from each color class, based on an eight-point color scale that ranged from yellow to green. Sample sizes are noted above bars.

sons) of the median (of 100 individuals within a simulated season) and compared these values between the two strategies.

Adult survival probability, egg load, and the relative survival probability of larvae of indiscriminate vs. choosy females had the largest impacts on model outcomes. The adult survivorship function directly impacted time limitation, and the function with daily survival probability of 0.95 for the first week and 0.20 for the second week (adult survivorship II) resulted in a clear advantage of the choosy oviposition strategy (Fig. 9). Presumably, this was because survival probability remains high for a full week, allowing choosy individuals to lay most of their eggs prior to death, despite their slow rate of oviposition. Lower egg loads also decreased the relative impact of time limitation and increased the advantage of choosy vs. indiscriminate strategies (Fig. 9).

The simulations did not provide evidence for a general negative impact of choosiness on female reproductive success and are suggestive of an overall advantage. Only in the most time-limited simulations (adult survivorship I, egg load $=100$ ) with the highest ratio of indiscriminate to choosy survival, did the indiscriminate strategy result in a noticeable advantage in reproductive success; and even with these parameters, the comparison of paired females within a simulated flight season indicated that choosiness was the better strategy an average of $37 \%$ of the time (Fig. 9a). When adult survivorship II was used, the choosy strategy always resulted in greater reproductive output and was favored in $48 \%$ to $73 \%$ of the pairwise comparisons (Fig. 9b). Finally, if we considered the mean value for relative larval survival of indiscriminate vs. choosy strategies $(0.56)$, the two strategies were very similar in success under adult survivorship I, while the choosy strategy had a clear advantage with adult survivorship II (Fig. 9).

\section{Discussion}

The adult activity and larval development of many species of butterflies are tightly constrained by environmental conditions and host plant phenology. Reproductive success is often limited by oviposition opportunities rather than egg production. Dempster (1983) concluded that failure to lay a full egg complement significantly impacted populations of 8 out of the 14 butterfly species reviewed. Butterflies that lay their eggs singly may suffer the largest impacts due to the greater time investment per egg. Courtney (1984) estimated that, on average, just $17 \%$ of potential eggs were laid in seven single-laying species. Many Pierids require direct insolation for flight, and researchers have frequently suggested that realized fecundity may be limited by available flight time (Ives 1978, Jones 1977,


FIG. 7. (a) Reproductive success for 100 each of choosy and indiscriminate females during a single simulated flight season, and (b) median reproductive success for 2500 simulated flight seasons (seasons with median $=0$, included values $>0$ ). Model results are shown for simulations when egg load $=75 \mathrm{eggs} / \mathrm{female}$, daily adult survival probability is fixed at 0.8 (adult survivalship I; see Methods), and all other parameters are set to mean values (see Table 1). "Choosy" females display observed host plant discrimination when choosing sites to oviposit; "indiscriminate" females are hypothetical butterflies that oviposit on all investigated host plants. 


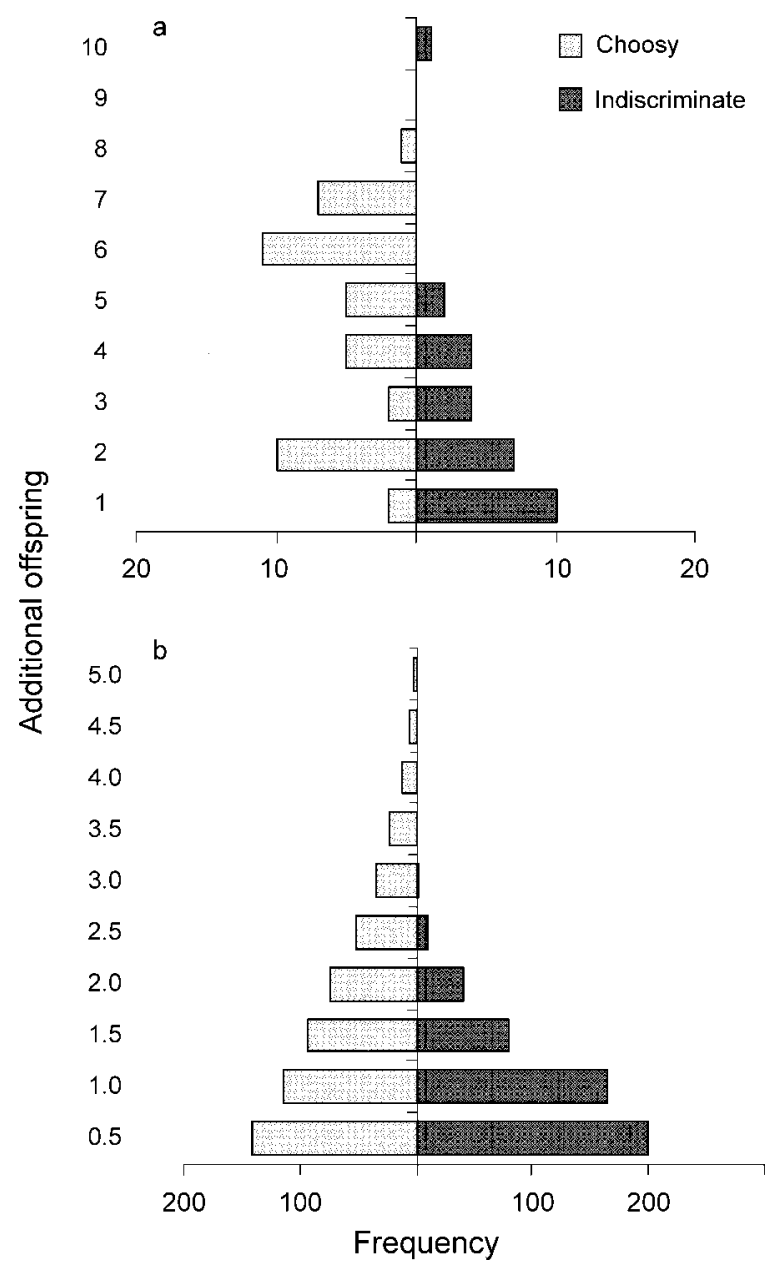

FIG. 8. The difference in offspring number between pairs of choosy and indiscriminate females experiencing the same simulated conditions (a) for 100 pairs during a single simulated flight season and (b) median of paired differences for each of 2500 simulated flight seasons. Thus, 10 indiscriminate females had one more offspring than their choosy counterparts, and in 150 simulated flight seasons, indiscriminate females had a median advantage of 0.5 offspring compared to choosy females. Parameter values and descriptions are as in Fig. 7.

1987, Jones and Ives 1979, Courtney and Duggan 1983, Kingsolver 1983, Courtney 1986).

Other species are most strongly impacted by host plant phenology. The impact of host plant senescence on larval survival and female reproductive success has been extensively documented for Euphydryas editha (e.g., Ehrlich et al. 1975, Weiss et al. 1988, Cushman et al. 1994, Fleishman et al. 1997, Boughton 1999), and the lycaenid Euphilotes enoptes is similarly constrained (Peterson 1997). For both species the problem of matching butterfly and host plant phenology can be ameliorated by topographic diversity.

Pieris virginiensis is tightly constrained by both environmental conditions suitable for flight and the phenology of its host plant. Host plant phenology is fairly constant between years, while $P$. virginiensis emergence and flight dates vary; this can lead to poor matching of butterfly and host phenology and reduced larval survival (Cappuccino and Kareiva 1985, and results herein). Unlike many species, $P$. virginiensis encounters little spatial heterogeneity in its mature hardwood habitat. Thus late-eclosing adults or those that have been grounded by poor weather are unlikely to find a distinct subset of host plants that will persist through larval development. While this might select for discrimination of individual host plants on the basis of senescence time, we found no evidence for this. These facts suggest that $P$. virginiensis may experience stronger time limitation than do many other species, and it is, in fact, widely cited as an example of a highly timelimited species (Shapiro 1971, Chew 1980, Cappuccino and Kareiva 1985, Shuey and Peacock 1989).

We found that the time available for flight and oviposition was both short and unpredictable on daily and seasonal scales. The flight season is just three weeks in length, and, on average, only $60 \%$ of days and $28 \%$ of daytime hours were suitable for flight. Furthermore, eggs laid earlier in the season had a marked survival advantage (2.4 and 4.6 times better in 1987 and 1988, respectively), because the larvae were more likely to complete development prior to host plant senescence. Yet, ovipositing females passed up approximately half of the host plants that they stopped to investigate and completely bypassed a great many more host individuals. This level of preference is especially interesting, given that Chew (1980) found no host species preference for $P$. virginiensis offered $D$. diphylla and three crucifers that it would not encounter in its woodland habitat, and Shapiro (1971) notes that under artificial conditions, it oviposits and successfully develops on a variety of crucifers.

At first, this level of choosiness might appear a poor strategy for time-limited individuals, and, in fact, this was the idea that led to the plant choice experiment. Being choosy can carry two obvious costs. It increases the total time required for oviposition, thereby decreasing the chance that a female will lay all of her eggs before she either reaches the end of the suitable flight season or dies. In addition, a cost may be incurred by her offspring in the form of reduced survival of eggs laid later in the season. For our population of $P$. virginiensis, these costs came with a striking benefit. Those plants chosen for oviposition provided an almost two-fold survival advantage for first- to fourth-instar larvae compared with unchosen neighbors of similar size.

Time-limited species are predicted to engage in relatively low levels of host plant discrimination (Levins and MacArthur 1969, Jaenike 1978, Courtney 1982, Chew and Robbins 1984, Mangel 1987). A mismatch between oviposition behavior and local conditions could occur if the population was genetically constrained in this adaptation, in the process of adapting 


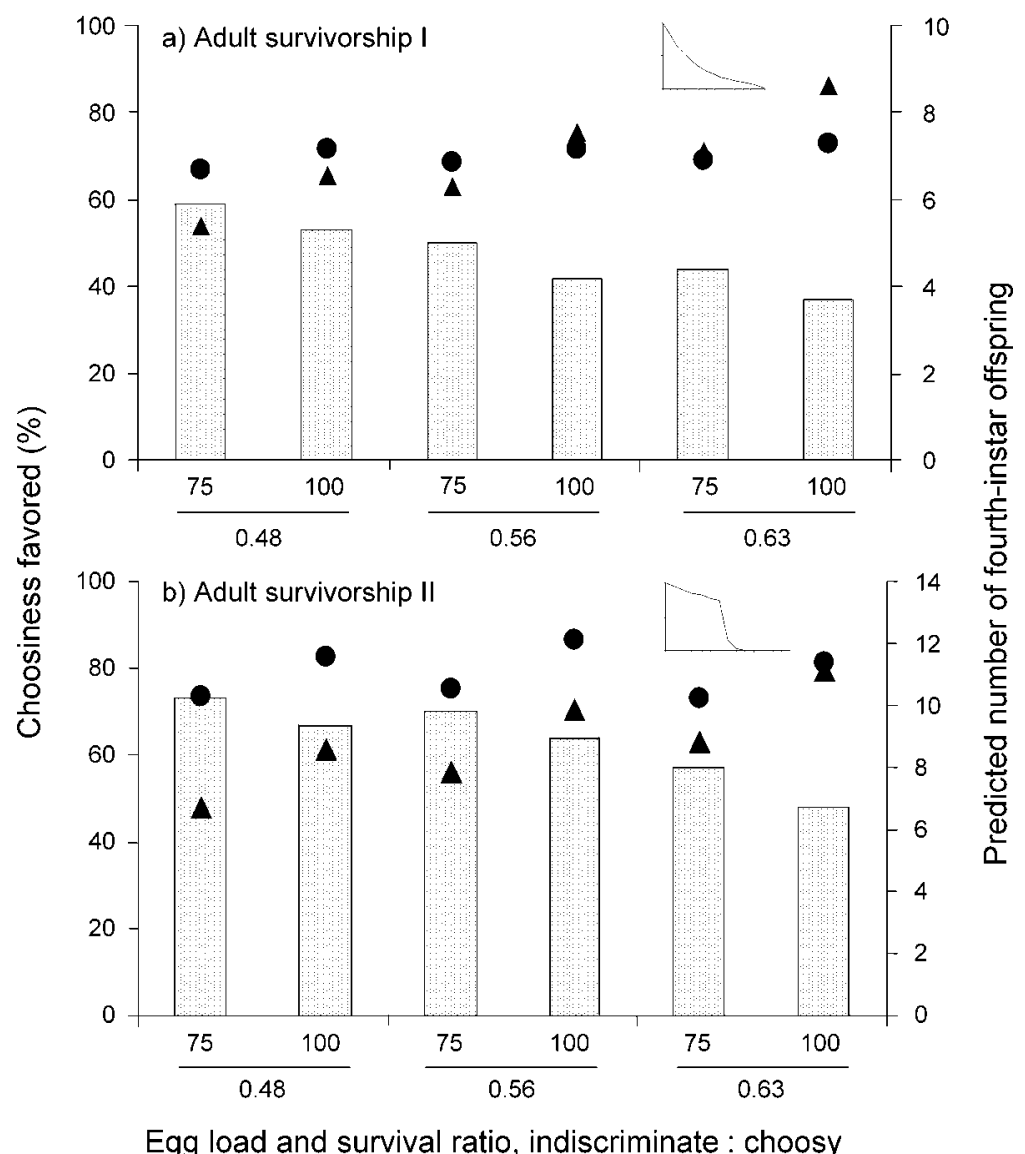

FIG. 9. The percentage of the 2500 simulated flight seasons in which the choosy strategy (circles) fared better than the indiscriminate strategy (triangles) and the mean (over 2500 simulated flight seasons) of the median values (for each flight season) for predicted number of fourth-instar offspring. Results are shown for combinations of the two survivorship curves, loads of 75 and 100 eggs per female, and three values for the relative first- to fourth-instar survival probability of indiscriminate compared to choosy strategies.

to local conditions, or diluted by immigration from less time-limited populations. Given the small size of the studied population (Cappuccino and Kareiva 1985), maintenance through immigration seems plausible, although $P$. virginiensis' apparent disinclination to cross open fields (Cappuccino and Kareiva 1985) could seriously undermine dispersal.

Furthermore, the large temporal variability in conditions suitable for oviposition and larval development create a classic scenario for selection of a bet-hedging strategy, where variance in fitness is reduced at the cost of lower arithmetic mean fitness, but with the benefit of increased geometric mean fitness (Philippi and Seger 1989, Hopper 1999). When compared to the observed level of host discrimination, our "indiscriminate" oviposition behavior represents a bet-hedging strategy. Yet, our simulation models did not reveal any clear disadvantage to choosiness, and they suggest that it is a favored strategy much of the time. While the hypothetical indiscriminate oviposition behavior used in our simulation decreased the variance in reproductive success, it did not lead to a substantial or consistent ad- vantage in geometric mean fitness. The two strategies fared more similarly under poor conditions and differed most under favorable conditions, where choosy individuals displayed a net advantage. One caveat is that we likely shortchanged the indiscriminate females. They oviposited on plants that were inspected by females, but were assigned the larval survival of the unchosen plants from the plant choice experiment. If an initial level of discrimination occurs when females decide to investigate a plant, these plants may confer higher survivorship than did our haphazard sample of plants without eggs. On the other hand, our haphazard sample likely included some high-quality hosts that by chance escaped oviposition.

We also observed a great deal of variability in choosiness. Variability could result from fluctuating selection in years with more or less favorable flight conditions. However, the observed variability is just as likely due to changing strategies as individuals age and/or decline in egg load. Models predict that, as individuals age and become more time limited, they may display decreased discrimination; on the other hand, as an individual's 
egg load declines, discrimination may increase (Jaenike 1978, Courtney 1982, Mangel 1987, Courtney et al. 1989). As egg load and available oviposition time decrease in concert, the changing balance of their effects is not clear.

Our research suggests that, despite the constraints of poor spring weather, overstory leaf-out, and the rapid senescence of Dentaria diphylla, behavior that results in the bypassing of the vast majority of host plant ramets aids not only larval survival but also adult female fitness. It is likely that populations with oviposition strategies primarily influenced by time limitation experience exacerbating impacts of widely dispersed host plants, high adult predation pressure, and/or other demands on the female's time, such as feeding. The relative impacts of time vs. egg limitation are also important. A number of studies suggest that $P$. rapae populations employ oviposition strategies driven by time limitation (Ives 1978, Jones and Ives 1979, Jones 1977, 1987). For instance, $P$. rapae shows relatively little host discrimination and does not widely spread its eggs in British Columbia and the UK when compared to Australian populations; the possibility that this is due to poor weather conditions seems more plausible when we consider that it can have 500-1000 eggs (Courtney 1986, Chew 1995). This may result in an oviposition strategy more strongly influenced by time rather than egg limitation. When we consider $P$. virginiensis in this context, it is noteworthy that it has a low egg load compared to many species. Given how few eggs she has, a $P$. virginiensis female may face stronger egg than time limitation and benefit by investing in careful placement of her eggs.

Oviposition behavior will be impacted by latency during successive egg maturation and dispersal behavior, as well as host preference. While some latency likely occurs, it is unlikely to explain the observed tendency to bypass many host plants; we assume that a female does not investigate host individuals for potential oviposition until she is ready to oviposit, and we often observed sequences where a female investigated a host, but did not oviposit, and then flew above and bypassed other hosts before again investigating one for oviposition. Cappuccino and Kareiva (1985) found that $P$. virginiensis follow linear flight paths fitting an "egg-spreading syndrome" (Root and Kareiva 1984). This may contribute to their propensity to bypass host plants without investigation, but our finding of higher survival on chosen hosts strongly suggests that females are also discriminating on the basis of plant or microhabitat characteristics.

Unfortunately, we do not know the discrimination cue or the mechanisms by which higher larval performance is conferred. Females did not choose plants that senesced later, although larvae on such plants gained a survival benefit. Cappuccino and Kareiva (1985) found that oviposition did not differ between ramets of different sizes despite poor searching abilities of larvae forced to move between plants. We failed to find any relationship between larval survival and host plant density or watering, suggesting that these are likely not providing cues for oviposition choices. Larval mortality may be strongly impacted by disease and natural enemies. Cappuccino and Kareiva (1985) documented that $22 \%$ of observed $P$. virginiensis larvae died of granulosis virus, and predators and parasitoids are recognized as important mortality factors for many Lepidoptera (Dempster 1983). Egg-spreading behavior may decrease the risks associated with localized disease contamination (Cappuccino and Kareiva 1985) or pockets of high predation. It is also possible that $P$. virginiensis is able to distinguish and chose host plants or microhabitats on the basis of these mortality factors.

This research demonstrates that even when time for flight and larval development are extremely time limited and variable, a high degree of host plant discrimination may be favored. We extend the finding of concordance between female oviposition preference and larval performance to examine the net impact on female fitness. In this case, it appears that what is best for individual larvae is also likely to be best for the mother. Future studies directly linking the choosiness of individual females to their resulting fitness or comparing choosiness between populations with different levels of time limitation would increase our ability to evaluate the costs and benefits of host-choice behavior.

\section{ACKNOWLEDGMENTS}

We thank P. Marino, W. F. Morris, P. Turchin, and many others for their help in the field, and D. F. Doak, M. F. Morris, and participants in the UAF EE Discussion group for their advice. The manuscript also benefited from the suggestions of D. Wagner, A. Watson, and two anonymous reviewers. Frances Chew generously provided us with unpublished information on $P$. virginiensis biology. The New Haven Water Company allowed us to use their lands. Funding was through NSF grant number 8605303 to P. Kareiva and J. Kingsolver.

\section{Literature Cited}

Baker, S. C., and S. G. Candy. 2002. Comparison of feeding efficiency, development time and survival of Tasmanian eucalyptus leaf beetle larvae Chrysophtharta bimaculata (Olivier) (Coleoptera: Chrysomelidae) on two hosts. Australian Journal of Entomology 41:174-181.

Ballabeni, P., M. Wlodarczyk, and M. Rahier. 2001. Does enemy-free space for eggs contribute to a leaf beetle's oviposition preference for a nutritionally inferior host plant? Functional Ecology 15:318-324.

Boughton, D. A. 1999. Euphydryas editha population extinctions due to unusual frosts. Ecology 80:2727-2739.

Cappuccino, N., and P. Kareiva. 1985. Coping with a capricious environment: a population study of a rare pierid butterfly. Ecology 66:152-161.

Carr, T. G., H. Roininen, and R. W. Price. 1998. Oviposition preference and larval performance of Nematus oligospilus (Hymenoptera: Tenthredinidae) in relation to host plant vigor. Environmental Entomology 27:615-625.

Chew, F. S. 1980. Foodplant preferences of Pieris caterpillars (Lepidoptera). Oecologia 46:347-353.

Chew, F. S., and R. K. Robbins. 1984. Egg-laying in butterflies. Pages 65-79 in R. I. Vane-Wright and P. R. Ackery, editors. The biology of butterflies. Academic Press, London, UK. 
Courtney, S. P. 1982. Coevolution of pierid butterflies and their cruciferous foodplants. V. Habitat selection, community structure and speciation. Oecologia 54:101-107.

Courtney, S. P. 1984. The evolution of batch oviposition by Lepidoptera and other insects. American Naturalist 123: 276-281.

Courtney, S. P. 1986. The ecology of pierid butterflies: dynamics and interactions. Advances in Ecological Research 15:51-131.

Courtney, S. P., G. K. Chen, and A. Gardner. 1989. A general model for individual host selection. Oikos 55:55-65.

Courtney, S. P., and A. Duggan. 1983. The population of the orange-tip butterfly, Anthocharis cardamines in Britain. Ecological Entomology 8:271-281.

Craig, T. P., J. K. Itami, C. Shantz, W. G. Abrahamson, J. D. Horner, and J. V. Craig. 2000. The influence of host plant variation and intraspecific competition on oviposition preference and offspring performance in the host races of $E \mathbf{~}$ rosta solidaginis. Ecological Entomology 25:7-18.

Cronin, J. T., and W. G. Abrahamson. 2001. Goldenrod stem galler preference and performance: effects of multiple herbivores and plant genotypes. Oecologia 127:87-96.

Cronin, J. T., W. G. Abrahamson, and T. P. Craig. 2001. Temporal variation in herbivore host-plant preference and performance: constraints on host-plant adaptation. Oikos 93: 312-320.

Cushman, J. H., C. L. Boggs, S. B. Weiss, D. D. Murphy, A. W. Harvey, and P. R. Ehrlich. 1994. Estimating female reproductive success of a threatened butterfly: influence of emergence time and host plant phenology. Oecologia 99: 194-200.

Dempster, J. P. 1983. The natural control of populations of butterflies and moths. Biology Review 58:461-481.

Ehrlich, P. R., R. R. White, M. C. Singer, S. W. McKechnie, and L. E. Gilbert. 1975. Checkerspot butterflies: a historical perspective. Science 188:221-228.

Fleishman, E., A. E. Launer, S. B. Weiss, J. M. Reed, C. L. Boggs, D. D. Murphy, and P. R. Ehrlich. 1997. Effects of microclimate and oviposition timing on prediapause larval survival of the Bay checkerspot butterfly, Euphydryas editha bayensis (Lepidoptera: Nymphalidae). Journal of Research on the Lepidoptera 36:31-44.

Fletcher, B. S., and R. J. Prokopy. 1991. Host location and oviposition in tephritid fruit files. Pages 139-171 in W. J. Bailey and J. Ridsdill-Smith, editors. Reproductive behaviour of insects. Individuals and populations. Chapman and Hall, London, UK.

Forsberg, J. 1987. Size discrimination among conspecific hostplants in two pierid butterflies; Pieris napi L. and Pontia daplidice L. Oecologia 72:52-57.

Fritz, R. S., B. A. Crabb, and C. G. Hochwender. 2000. Preference and performance of a gall-inducing sawfly: a test of the plant vigor hypothesis. Oikos 89:555-563.

Hilborn, R., and M. Mangel. 1997. The ecological detective: confronting models with data. Princeton University Press, Princeton, New Jersey, USA.

Hopper, K. R. 1999. Risk-spreading and bet-hedging in insect population biology. Annual Review of Entomology 44: 535-560.

Ives, P. M. 1978. How discriminating are cabbage butterflies? Australian Journal of Ecology 3:261-276.

Jaenike, J. 1978. On optimal oviposition behaviour by phytophagous insects. Theoretical Population Biology 14:350356.

Joachim-Bravo, I. S., O. A. Fernandes, S. A. De Bortoli, and F. S. Zucoloto. 2001. Oviposition Behavior of Ceratitis capitata Wiedemann (Diptera: Tephritidae): association between oviposition preference and larval performance in individual females. Neotropical Entomology 30:559-564.
Jones, R. E. 1977. Movement patterns and egg distribution of cabbage butterflies. Journal of Animal Ecology 46:195212.

Jones, R. E. 1987. Behavioural evolution in the cabbage butterfly (Pieris rapae). Oecologia 72:69-76.

Jones, R. E., and P. M. Ives. 1979. The adaptiveness of searching and oviposition behaviour in Pieris rapae L. Australian Journal of Ecology 46:195-212.

Kauffman, M. J., J. F. Pollock, and B. Walton. 2004. Spatial structure, dispersal, and management of a recovering raptor population. American Naturalist 164:582-597.

Kessler, A., and I. T. Baldwin. 2002. Manduca quinquemaculata's optimization of intra-plant oviposition to predation, food quality, and thermal constraints. Ecology 83: 2346-2354.

Kingsolver, J. G. 1983. Ecological significance of flight activity in Colias butterflies: implications for reproductive strategy and population structure. Ecology 64:546-55.

Kingsolver, J. G. 1988. Thermoregulation, flight, and the evolution of wing pattern in pierid butterflies: the topography of adaptive landscapes. American Zoologist 28:899912.

Levins, R., and R. MacArthur. 1969. An hypothesis to explain the incidence of monophagy. Ecology 50:910-911.

Macel, M., P. I. Klinkhamer, K. Vrieling, and E. van der Meijden. 2002. Diversity of pyrrolizidine alkaloids in $\mathrm{Se}$ necio species does not affect the specialist herbivore Tyria jacobaeae. Oecologia 133:541-550.

Mangel, M. 1987. Oviposition site selection and clutch size in insects. Journal of Mathematical Biology 25:1-22.

Mayhew, P. J. 1997. Adaptive patterns of host-plant selection by phytophagous insects. Oikos 79:417-428.

Peterson, M. A. 1997. Host plant phenology and butterfly dispersal: causes and consequences of uphill movement. Ecology 78:167-180.

Philippi, T., and J. Seger. 1989. Hedging one's evolutionary bets, revisited. Trends in Ecology and Evolution 4:41-44.

Pierce, N. E., M. F. Braby, A. Heath, D. J. Lohman, J. Mathew, D. B. Rand, and M. A. Travassos. 2002. The ecology and evolution of ant association in the Lycaenidae (Lepidoptera). Annual Review of Entomology 47:733-771.

Price, P. W. 1984. Insect ecology. Second edition. John Wiley and Sons, New York, New York, USA.

Rausher, M. D. 1979. Egg recognition: its advantage to a butterfly. Animal Behavior 27:1034-1040.

Root, R. B., and P. M. Kareiva. 1984. The search for resources by cabbage butterflies (Pieris rapae): ecological consequences and adaptive significance of Markovian movements in a patchy environment. Ecology 65:147-165.

Scheirs, J., and L. De Bruyn. 2002. Integrating optimal foraging and optimal oviposition theory in plant-insect research. Oikos 96:187-191.

Scheirs, J., L. De Bruyn, and R. Verhagen. 2000. Optimization of adult performance determines host choice in a grass miner. Proceedings of the Royal Society London, Series B 267:2065-2069.

Scheirs, J., T. G. Zoebisch, D. J. Schuster, and L. De Bruyn. 2004. Optimal foraging shapes host preference of a polyphagous leafminer. Ecological Entomology 29:375-379.

Shapiro, A. M. 1971. Occurrence of a latent polyphenism in Pieris virginiensis (Lepidoptera: Pieridae). Entomology News 82:13-16.

Shapiro, A. M. 1979. The phenology of Pieris napi microstriata during and after the 1975-77 California drought, and its evolutionary significance. Psyche 86:8-10.

Shiojiri, K., and J. Takabayashi. 2003. Effects of specialist parasitoids on oviposition preference of phytophagous insects: encounter-dilution effects in a tritrophic interaction. Ecolological Entomology 28:573-578. 
Shuey, J. A., and J. W. Peacock. 1989. Host plant exploitation by an oligophagous population of Pieris virginiensis (Lepidoptera: Pieridae). American Midland Naturalist 122: 255261.

Singer, M. C. 1984. Butterfly-host plant relationships: host quality, adult choice and larval success. Pages 81-90 in R. I. Vane-Wright and P. R. Ackery, editors. The biology of butterflies. Academic Press, London, UK.

Solarz, S. L., and R. M. Newman. 2001. Variation in host plant preference and performance by the milfoil weevil, Euhrychiopsis lecontei Dietz, exposed to native and exotic watermilfoils. Oecologia 126:66-75.

Strong, D. R., J. H. Lawton, and Sir R. Southwood. 1984. Insects on plants: community patterns and mechanisms. Harvard University Press, Cambridge, Massachusetts, USA.
Thompson, J. N. 1988. Evolutionary ecology of the relationship between oviposition preference and performance of offspring in phytophagous insects. Entomologia Experimentalis et Applicata 47:3-14.

Van Nouhuys, S., M. C. Singer, and M. Nieminen. 2003. Spatial and temporal patterns of caterpillar performance and the suitability of two host plant species. Ecological Entomology 28:193-202.

Weiss, S. B., D. D. Murphy, and R. R. White. 1988. Sun, slope, and butterflies: topographic determinants of habitat quality for Euphydryas editha. Ecology 69:1486-1496.

Wise, M. J., and A. M. Weinberg. 2002. Prior flea beetle herbivory affects oviposition preference and larval performance of a potato beetle on their shared host plant. Ecological Entomology 27:115-122. 